\title{
Two-dimensional phase transformation probed by second harmonic generation: Oscillatory transformation of the K/Al(111) system
}

\author{
Z. Charles Ying and E. Ward Plummer* \\ Oak Ridge National Laboratory, P. O. Box 2008, Oak Ridge, Tennessee 37831 \\ *also at Department of Physics and Astronomy, University of Tennessee, Knoxville, Tennessee 37996
}

To be published in Laser Techniques for Surface Science II edited by J. M. Hicks, W. Ho, and H.-L. Dai

Proceedings of SPIE's International Symposium on Optical Science, Engineering, and Instrumentation Volume 2547 (1995)

\section{DISCLAIMER}

\begin{abstract}
This report was prepared as an account of work sponsored by an agency of the United States Government. Neither the United States Government nor any agency thereof, nor any of their employees, makes any warranty, express or implied, or assumes any legal liability or responsibility for the accuracy, completeness, or usefulness of any information, apparatus, product, or process disclosed, or represents that its use would not infringe privately owned rights. Reference herein to any specific commercial product, process, or service by trade name, trademark, manufacturer, or otherwise does not necessarily constitute or imply its endorsement, recommendation, or favoring by the United States Government or any agency thereof. The views and opinions of authors expressed herein do not necessarily state or reflect those of the United States Government or any agency thereof.
\end{abstract}

Research sponsored by the Office of Health and Environmental Research, U. S. Department of Energy, under contract DE-AC05-840R21400 with Lockheed Martin Energy Systems, Inc.

"The submitted manuscript has been authored by a contractor of the U.S. Government under contract DE-AC05-84OR21400. Accordingly, the U. S. Government retains a nonexclusive, royalty-free license to publish or reproduce the published form of this contribution, or allow others to do so, for U. S. Government purposes." 


\title{
Two-dimensional phase transformation probed by second harmonic generation: Oscillatory transformation of the $\mathrm{K} / \mathrm{Al}(111)$ system
}

\author{
Z. Charles Ying and E. Ward Plummer* \\ Oak Ridge National Laboratory, P. O. Box 2008, Oak Ridge, Tennessee 37831 \\ *also at Department of Physics and Astronomy, University of Tennessee, Knoxville, Tennessee 37996
}

\begin{abstract}
The technique of optical second harmonic generation is used to study phase transformations at two-dimensional surfaces and interfaces. Examples are given to illustrate that changes in surface symmetry, adsorption configuration, and electronic structure can be detected by this nonlinear optical technique. An oscillatory phase transformation of potassium adsorbed atoms on $\mathrm{Al}(111)$ probed by second harmonic generation is analyzed in detail.
\end{abstract}

Keywords: nonlinear optics, second harmonic generation, phase transformation, surface, alkali metal, aluminum

\section{INTRODUCTION}

The optical second harmonic generation (SHG) technique has been employed in a number of surface and interface studies. Because this pure-optical technique does not involve an electron or ion beam, SHG can be used in investigation of liquid surfaces, buried solid interfaces in addition to solid surfaces kept in ultrahigh vacuum (UHV). The temporal resolution of the SHG technique is restricted only by the pulse width of the excitation laser. Ultrafast time resolution in femtoseconds has been achieved. 1, 2, 3 The spatial resolution of SHG is limited by diffraction. Surface imaging using the SHG process with a $5-\mu \mathrm{m}$ lateral resolution has been demonstrated recently. ${ }^{4}$ There are no major obstacles to achieve submicrometer resolution. Using a circularly polarized laser beam, magnetic materials 5,6 and adsorption of chiral molecules ${ }^{7}$ can also be studied.

Most surface SHG studies performed up to date are concentrated on adsorption and desorption of atoms or molecules. In these studies, the changes of adsorbate coverage with exposure or heating are monitored by SHG. In this paper, we will discuss the application of the SHG technique to study surface phase transformations. The chief advantage of the SHG technique is that the phase transformations can be monitored in real time. Phase transformations with either geometric or electronic changes can be probed. In the following sections, we will first review the basic principles of the SHG process and the current status of research on its application in studying surface phase transformations, which will be followed by a detailed analysis of a phase transformation of potassium on an $\mathrm{Al}(111)$ surface, where the adsorbate changes between two adsorption configurations in an oscillatory fashion as temperature increases. ${ }^{8}$ 


\section{BASICS OF SECOND HARMONIC GENERATION}

The response of a material under photon excitation, as measured by polarization $\mathbf{P}$, is usually described by a linear relationship with the electric field of the radiation, $\mathbf{E}$. Under irradiation of an intense laser, however, the higher-order terms can no longer be neglected so that

$$
\mathbf{P}=\chi^{(1)} \mathbf{E}+\chi^{(2)} \mathbf{E E}+\chi^{(3)} \mathbf{E E E}+\cdots .
$$

The SHG process is described by the second-order nonlinear susceptibility $\chi^{(2)}$. Under the electric-dipole approximation, $\chi^{(2)}$ vanishes in the bulk of a material with an inversion symmetry, as is the case for many metals as well as elementary semiconductors. Only much weaker contributions such as magnetic dipole and electric quadrupole exist for the SHG process in the bulk. At a surface (or interface in general), the inversion symmetry is broken and an electric-dipole contribution to SHG is allowed. This surface electric-dipole term is often found to dominate the whole SHG signal and varies sensitively to changes in surface electronic and geometric structures. That is why the SHG technique can be used for surface studies.

Because $\chi^{(2)}$ is a tensor of $3 \times 6=18$ elements, the second harmonic signal generated from a crystalline surface varies as one rotates the azimuthal angle of the crystal. 9 The pattern of the azimuthal angle dependence is different for different faces. This is true even for cubic materials, where the linear optical response from the bulk is always isotropic. For example, the azimuthal angle dependence of the SHG signal from a (111) surface of a cubic crystal, which has a three-fold symmetry, exhibits a three-fold symmetry pattern. Specifically, under $p$-polarized photon excitation, the $p$-polarized second harmonic signal generated from the (111) surface has the form of

$$
I_{\mathrm{p}}(2 \omega)=|A+C \cos (3 \phi)|^{2} E_{\mathrm{p}}^{4}(\omega),
$$

while the $s$-polarized SHG is given by

$$
I_{\mathrm{s}}(2 \omega)=|B \sin (3 \phi)|^{2} E_{\mathrm{p}}^{4}(\omega),
$$

where the azimuthal angle $\phi$ defines to be zero in the [2 $\overline{1} \overline{1}]$ direction. If the surface symmetry is changed during a phase transformation, it will be reflected in the azimuthal angle dependence of the SHG signal and therefore be detected.

Another way to study surface phase transformations by SHG is to measure an azimuthally isotropic second harmonic current at the surface, such as the one that is associates with the $A$ term in Eq. (2). Direct measurement of this $A$ term can be made with the choice of an azimuthal angle $\phi=30^{\circ}$. The dominant contribution to $A$ is a nonlinear current perpendicular to the surface excited by the perpendicular electric field. This perpendicular current is associated with the discontinuity of the macroscopic electric field in the perpendicular direction. Its microscopic origin is the dynamic screening of surface electrons. ${ }^{10}$ The magnitude of this nonlinear current is very sensitive to the surface conditions. Changes on several orders of magnitude in SHG intensity have been observed with adsorption of a submonolayer of alkali-metal atoms on metal surfaces. ${ }^{8,11}$ Such high a sensitivity allows easy detection surface phase transformations. 
A nonlinear isotropic current parallel to the surface, excited by photons of both $s$ and $p$ polarizations, can also be measured experimentally. On a (100) surface of a cubic material, for example, the $s$-polarized SHG intensity is given by

$$
I_{\mathrm{s}}(2 \omega)=\left|[D-F+F \cos (4 \phi)] E_{\mathrm{s}}(\omega) E_{\mathrm{p}}(\omega)+\left[G E_{\mathrm{s}}^{2}(\omega)+H E_{\mathrm{p}}^{2}(\omega)\right] \sin (4 \phi)\right|^{2},
$$

where $D$ is associated with the parallel isotropic current. Terms $F, G$, and $H$ are related to a single SHG source: the anisotropic contribution from the bulk, which is often weak. (Additional SHG sources are present at other faces due to lower symmetries.) It can be expected that the parallel current is large for a metallic surface and small for an insulating surface. Therefore, SHG can be used to measure metalinsulator transitions. ${ }^{12}$

\section{PREVIOUS SHG STUDIES OF SURFACE TRANSFORMATIONS}

As discussed above, surface phase transformations that are accompanied by changes of surface symmetry can be detected by SHG measurement as a function of azimuthal angle. An early experimental study using this idea was due to Heinz et al. They have measured SHG signal for Si(111) surface in UHV at both $2 \times 1$ and $7 \times 7$ reconstructions. ${ }^{13}$ The two reconstruction structures of the Si surface have different symmetries. Very different SHG patterns with respect to the azimuthal angle have been observed.

Transient phenomena at surfaces can also be probed by SHG. In an experiment by Shank et al., a high-intensity femtosecond-laser pulse was impinged on a Si(111) surface. ${ }^{1}$ The disordering and melting of the surface atoms after irradiation of this high-intensity pulse was monitored by SHG excited by a second and weaker femtosecond-laser pulse at different delay times. Before irradiation of the highintensity pulse, the SHG exhibited a 3-fold azimuthal-angle pattern that reflects the symmetry of the $\mathrm{Si}(111)$ surface. At a delay of $240 \mathrm{fs}$ after the high-intensity pulse, the azimuthal-angle dependence becomes much weaker, suggesting disorder of the surface atoms. The SHG pattern became isotropic after a delay of $3 \mathrm{ps}$, which indicates melting of the surface layer. This experiment has demonstrated the powerfulness of the SHG technique in detecting ultrafast changes occurring at surfaces. A similar experimental study, with higher time resolution, was reported by Tom et $_{\text {al. }}{ }^{2}$

A recent SHG study utilized the fact that a nonlinear current parallel to the surface can be probed by s-polarized SHG with excitation of mixed polarizations. ${ }^{12}$ The phase of the second harmonic wave relative to the fundamental wave, in additional to the SHG intensity, was measured. On a $\mathrm{Si}(100)$ surface, the SHG in this polarization configuration did not change appreciably with adsorption of alkalimetal atoms up to $0.17 \mathrm{ML}$. After that, the phase of the second harmonic wave changed abruptly, which was accompanied by a large increase of the SHG intensity. These changes have been attributed to an insulator-to-metal transition on the surface at about $1 / 6$ of the full alkali-metal coverage.

The SHG studies of phase transformations are not limited to solid surfaces in UHV. The technique is also applicable to other types of interfaces. For example, a study was conducted for $\mathrm{Ag}(111)$ surface under an electrochemical solution..$^{14}$ A reconstruction of the surface, with a reduction of the surface symmetry from $\mathrm{C}_{3 \mathrm{v}}$ to $\mathrm{C}_{3}$, at certain potential of the electrode was detected by an azimuthal-angle study of the SHG intensity. Another study was conducted for various amphilphile molecules at an air- 
aqueous interface. ${ }^{15}$ Abnormally large fluctuations in the SHG intensity observed for these systems are attributed to phase transformations associated with the orientation of these molecules.

\section{TWO PHASES OF POTASSIUM ADSORPTION ON Al(111)}

In the following sections, we will discuss the observation of a phase transformation of potassium atoms on $\mathrm{Al}(111)$. The adsorption of alkali metals on surfaces of this simple metal has played an important role in the development of theories of adsorption as well as theories of surface-electron response to an external probe. The electronic and geometric structures of alkali-metal adsorbed on aluminum surfaces have a number of interesting features.

Early studies using low-energy electron diffraction (LEED) have determined that adsorption of alkali-metal atoms on $\mathrm{Al}(111)$ yields a $\sqrt{3} \times \sqrt{3}$ structure at a coverage of $0.33 \mathrm{ML}$. [One monolayer (ML) is defined here by the number of the surface atoms at $\mathrm{Al}(111)$.] Because of the low solubility of alkali metals in aluminum and the close packing of the $\mathrm{Al}(111)$ surface, it was long believed that the alkali-metal atoms are adsorbed on top of the aluminum atoms, as shown in Fig. 1(a). A study a few years ago using surface extended $\mathrm{x}$-ray absorption fine structure (SEXAFS) has suggested, however, that sodium atoms are located in a substitutional configuration, as shown in Fig. 1(b), on $\mathrm{Al}(111)$ at room temperature. ${ }^{16}$ This unexpected result has been confirmed by $a b$ initio total-energy calculation ${ }^{17}$ and LEED-IV experiments ${ }^{18}$ It is now known that $\mathrm{K}$ and $\mathrm{Rb}$ atoms also adsorb substitutionally as they are deposited onto $\mathrm{Al}(111)$ at room temperature. ${ }^{19,20,21}$ The theory and LEED-IV experiments have also shown that the alkali-metal atoms are in the atop configuration on $\mathrm{Al}(111)$ at liquid nitrogen temperature and the transformation to the substitutional configuration is activated.

(a)

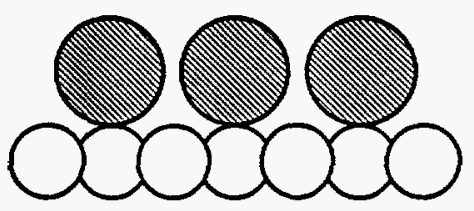

\section{Side View}

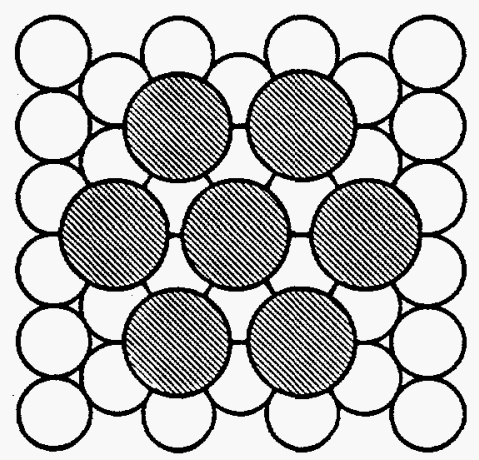

(b)
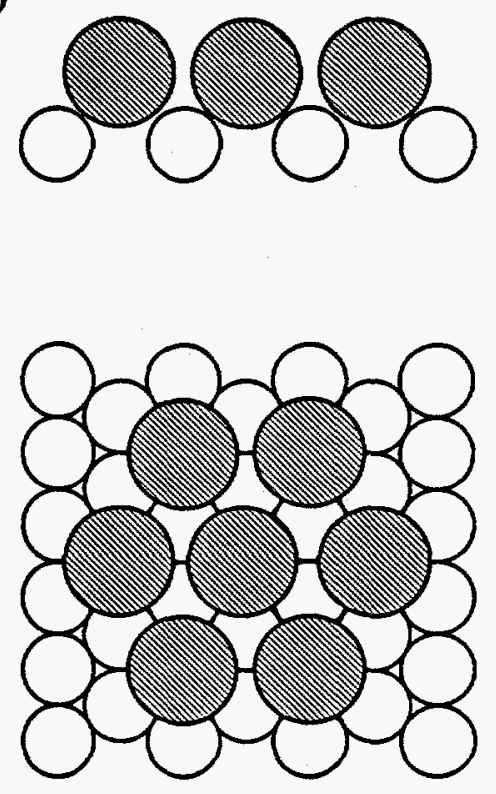

Top View

Fig. 1. Models for (a) the atop and (b) substitutional adsorption of alkali-metal atoms on $\mathrm{Al}(111)$. 
It is desirable to measure the temperature at which the alkali metal atoms transforms from the atop to substitutional configuration and characterize the phase transformation in detail. The techniques that have been used to differentiate the adsorption configuration of alkali-metal atoms on aluminum, including SEXAFS and LEED-IV, require long data-acquisition time and thus are not suitable for monitoring the phase transformation in real time. The data-acquisition time of a STM is much shorter, but STM usually operates only at room temperature. Second harmonic generation, on the other hand, can be used at any sample temperature and has a short data-acquisition time. (In our study, each data point represents counting for 3 seconds. The counting rate was $\sim 10^{2} / \mathrm{s}$ for the clean Al surface and up to $10^{5} / \mathrm{s}$ for the potassium-doped surface.) The different electronic structures corresponding to the two alkali-metal adsorption geometries on $\mathrm{Al}(111)$ are expected to gave different SHG efficiencies (see below).

\section{EXPERIMENTAL TECHNIQUE}

Our study of potassium adsorption and phase transformation on $\mathrm{Al}(111)$ was conducted in UHV. The Al(111) surface was cleaned by repeated circles of argon ion sputtering and annealing. The potassium was evaporated from an alkali-metal getter (SEAS). The coverage of potassium on the surface was calibrated by the work-function change, thermal desorption spectra, and LEED.

A mode-locked Nd:YAG laser (Quantronix 416) was used as the excitation source. The laser beam (1064 nm, $100 \mathrm{ps,} 76 \mathrm{MHz}$ ) was focused onto the surface with a beam diameter of $\sim 15 \mu \mathrm{m}$ at an incident angle $60^{\circ}$ from the surface normal. The second harmonic photons generated from the surface were separated from the fundamental beam by filters and counted by a photon counter. The data presented in this paper are recorded over a period of several minutes, when the surface was dosed with potassium atoms or heated by a dc current.

\section{PHASE TRANSFORMATION OF K ON AI(111) PROBED BY SHG}

The isotropic term of the $p$-polarized SHG signal excited by $p$-polarized photons, the $A$ term in Eq. (2), was chosen in studying the transformation of potassium atoms from the atop to substitutional configuration. As said earlier, this signal is mainly contributed by a nonlinear current perpendicular to the surface excited by the perpendicular component of the electric field. A jellium calculation under freeelectron approximation indicates that, for the excitation wavelength used in the experiment (1064 nm), this perpendicular current is near a resonant peak for the $\mathrm{K} / \mathrm{Al}(111)$ system. ${ }^{10}$ Since the atop adsorption configuration for potassium atoms on $\mathrm{Al}(111)$ more closely resembles a free-electron system, it is expected that the SHG intensity with the potassium atoms in the atop configuration will be significantly higher than in the substitutional configuration, where strong covalent bonding is predicted.

Figure 2 presents the temperature dependence of the $p$-polarized SHG intensity under $p$-polarized excitation for $\mathrm{Al}(111)$ covered with 0.24 and $0.33 \mathrm{ML}$ potassium. The data have been normalized to the value recorded at the clean $\mathrm{Al}(111)$ surface. As expected, the SHG intensity is high at low temperatures and decreases as the temperature increases, which reflects the phase transformation of adsorbed potassium atoms from the atop to substitutional configuration. The largest change occurs between 200 and $220 \mathrm{~K}$. Beyond $220 \mathrm{~K}$, the SHG intensity changes much slowly. It can be concluded that the phase transformation occurs near $200 \mathrm{~K}$ and completes by $220 \mathrm{~K}$. 



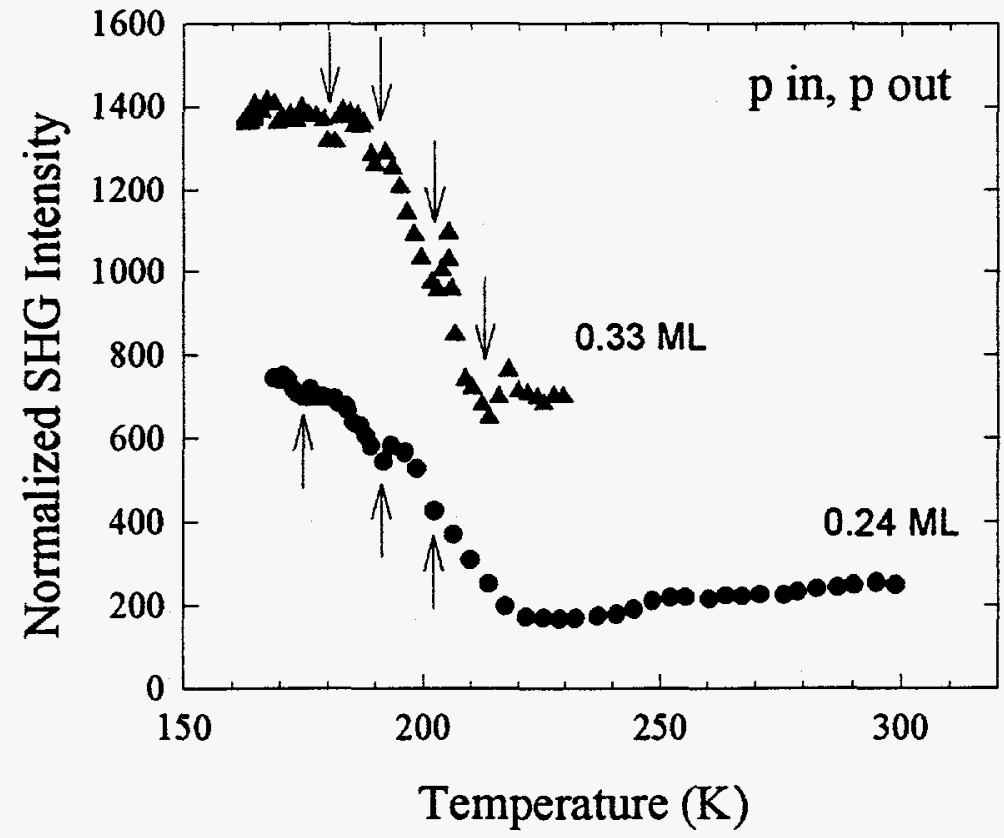

Fig. 2. The intensity of SHG from $\mathrm{Al}(111)$ deposited with 0.24 and $0.33 \mathrm{ML}$ potassium as a function of temperature. The data have been normalized to the SHG value for the clean surface.

It is noticed that, over a temperature range between 160 and $220 \mathrm{~K}$, the SHG intensity does not decrease monotonically. There exist several kinks, as marked by arrows in Fig. 2 . These kinks have deviations much larger than what would be predicted by statistical fluctuations and therefore are significant. Larger deviations are observed at $0.33 \mathrm{ML}$ than $0.24 \mathrm{ML}$.

The SHG intensity can be viewed as a sum of contributions from potassium atoms in the atop and substitutional configurations, with the latter contributing less on a per atom base. The presence of these kinks suggests that the transformation of potassium atoms does not proceeds smoothly. The effect can be seen more clearly for the data at $0.24 \mathrm{ML}$ in a plot of transformation rate calculated from the SHG data vs. temperature, ${ }^{8}$ as plotted in Fig. 3 . The transformation rate does not increases monotonically but oscillates as the temperature increases.

Oscillations in surface reactions have been observed previously for oxidation of $\mathrm{H}_{2}$ and $\mathrm{CO}$ on platinum surfaces. $^{22}$ In the present case, the feedback needed for the oscillation is the excess aluminum atoms on the islands of potassium atoms, produced as the potassium atoms changing into the substitutional configuration. The presence of a large number of these excess aluminum atoms should slow down and may even reverse momentarily the transformation. Eventually, these aluminum atoms are cleared out the potassium islands and probably attached to other aluminum atoms at step edges. The oscillation is therefore damped. In the case of $0.33 \mathrm{ML}$, the whole surface is covered with potassium atoms. It is more difficult for the excess aluminum atoms to escape the large islands, and consequently the 


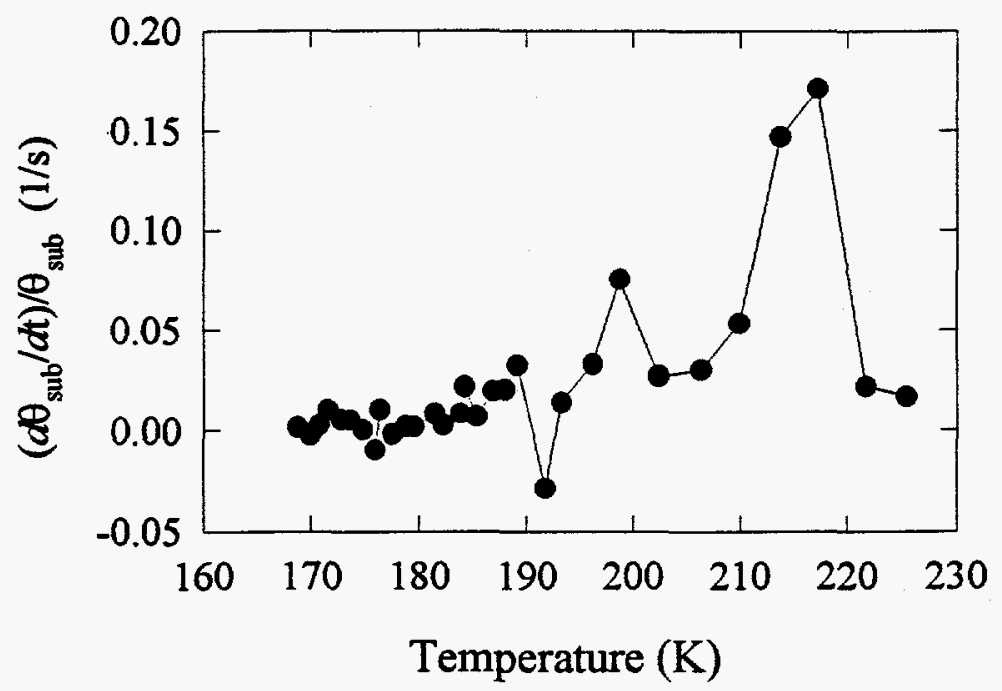

Fig. 3. The rate of potassium transformation from the atop to substitutional configuration for the surface with a total potassium coverage of $0.24 \mathrm{ML}$.

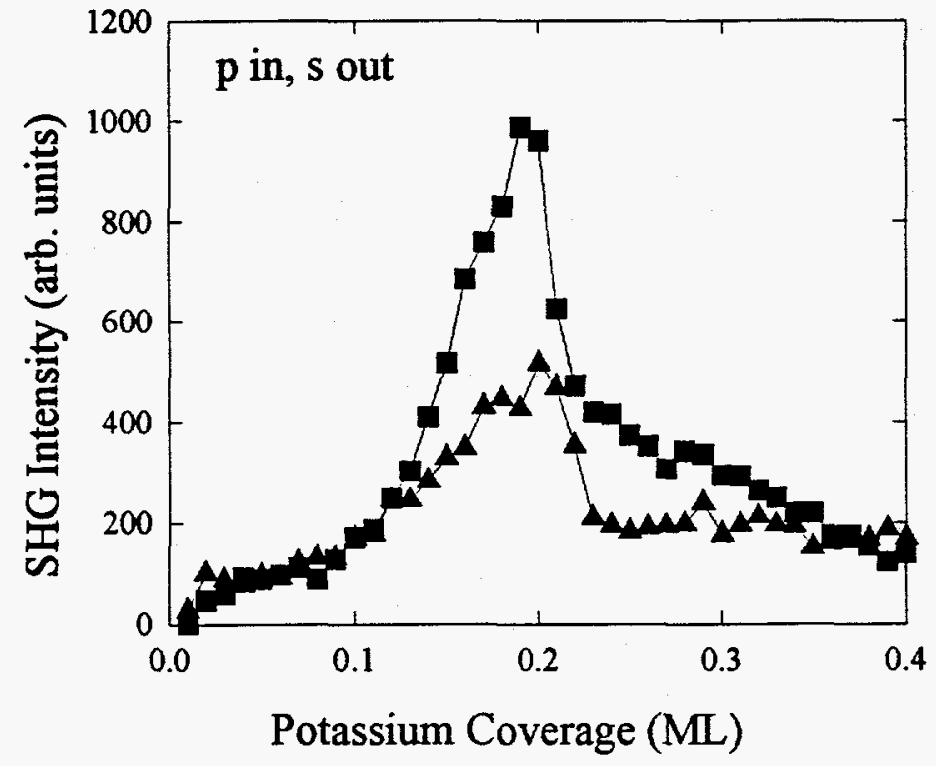

Fig. 4. Intensity of $s$-polarized SHG signal excited by p-polarized light as a function of potassium coverage. The azimuthal angles are $\phi=0^{\circ}$ (squares) and $30^{\circ}$ (triangles). 

damping is weak. That is why the oscillation amplitude are much larger at $0.33 \mathrm{ML}$ than $0.24 \mathrm{ML}$ (see Fig. 2). At a very low potassium coverage (e.g. $0.06 \mathrm{ML}$ ), potassium atoms do not form islands and there is no phase transformation during heating. ${ }^{8}$

Finally we will consider the order of the $\mathrm{Al}(111)$ surface covered with potassium atoms. An azimuthal angle dependence of $s$-polarized SHG signal from a perfect $\mathrm{Al}(111)$ surface should exhibit a three-fold symmetry and vanish at $\phi=0^{\circ}$ [see Eq. (3)]. This is exactly what has been observed for clean $\mathrm{Al}(111) .{ }^{23}$ Figure 4 shows $s$-polarized SHG signal from potassium-covered $\mathrm{Al}(111)$ surface at a temperature of $120 \mathrm{~K}$. Large SHG signal has been observed even at $\phi=0^{\circ}$, which indicates loss of surface order with potassium adsorption. For potassium coverages below $0.2 \mathrm{ML}$, the SHG intensity increases as the coverage increases, which reflects destroy of the surface order as more and more potassium atoms are adsorbed randomly on the surface. At $0.2 \mathrm{ML}$, the SHG intensity drops suddenly, indicating partial recover of the surface order. These data suggest that the randomly adsorbed potassium atoms are quickly organized and form large islands at $0.2 \mathrm{ML}$. Such a condensation phase transformation has been predicted theoretically. ${ }^{17}$

\section{CONCLUSIONS}

Optical second harmonic generation is a useful tool to study surfaces and interfaces. Being a pure-optical technique, it has many advantages, including environment flexibility, ultrahigh time resolution, and good spatial resolution. This paper shows that the SHG technique can be used to study phase transformations at surfaces and interfaces. Through several examples, it has been shown that the phase transformations with changes of interfacial symmetry can be revealed by an azimuthal-angle study of the SHG signal. Changes of electronic properties can be monitored by both the intensity and phase of the SHG signal that is associated with a nonlinear current either parallel or perpendicular to the surface.

The SHG technique is a versatile technique to study phase transformations at various surfaces and interfaces. In addition to the topic that have been mentioned in this paper, magnetic phase transformations can be observed using SHG with circularly polarized laser beams. 5,6 Adsorption of nanocluster materials on surfaces is another interesting subject. It is also possible to enhance the specificity of nonlinear optics by tuning the radiation to a resonant condition.

\section{ACKNOWLEDGMENTS}

The SHG research on $\mathrm{K} / \mathrm{Al}(111)$ reported in this paper was conducted in collaboration with Jian Wang and supported in part by National Science Foundation under Grant No. DMR93-96059 and the MRL Program at the University of Pennsylvania under Grant No. DMR91-20668. ZCY acknowledges receipt of a Eugene P. Wigner Fellowship at Oak Ridge National Laboratory.

\section{REFERENCES}

${ }^{1}$ C. V. Shank, R. Yen, and C. Hirlimann, Phys. Rev. Lett. 51, 900 (1983).

${ }^{2}$ H. W. K. Tom, G. D. Aumiller, and C. H. Brito-Cruz, Phys. Rev. Lett. 60, 1438 (1988).

3 J. A. Prybyla, H. W. K. Tom, and G. D. Aumiller, Phys. Rev. Lett. 68, 503 (1992).

${ }^{4}$ K. A. Schultz, I. I. Suni, and E. G. Seebauer, J. Opt. Soc. Am. B 10, 546 (1993). 
${ }^{5}$ J. Reif, C. Rao, and E. Matthias, Phys. Rev. Lett. 71, 2878 (1993).

${ }^{6}$ H. A. Wuerebga, M. W. J. Prins, D. L. Abraham, and Th. Rasing, Phys. Rev. B 50, 1282 (1994).

7 T. Petralli-Mallow, T. M. Wong, J. D. Byers, H. I. Lee, and J. M. Hicks, J. Phys. Chem. 97, 1383 (1993).

${ }^{8}$ J. Wang, Z. C. Ying, and E. W. Plummer, Phys. Rev. B 51, 5590 (1995).

9 J. E. Sipe, D. J. Moss, and H. M. van Driel, Phys. Rev. B 35, 1129 (1987).

${ }^{10}$ A. Liebsch, Phys. Rev. Lett. 67, 2858 (1991).

${ }^{11}$ H. W. K. Tom, et al., Surf. Sci. 172, 466 (1986).

${ }^{12}$ S. Arekat, S. D. Kevan, and G. L. Richmond, Europhys. Lett. 22, 377 (1993).

${ }^{13}$ T. F. Heinz, M. M. T. Loy, and W. A. Thompson, Phys. Rev. Lett. 54, 63 (1985).

${ }^{14}$ Y. Tang, L. J. Simpson, and T. E. Furtak, Phys. Rev. Lett. 67, 2814 (1991).

${ }^{15}$ X. Zhao and K. B. Eisenthal, J. Chem. Phys. 102, 5818 (1995).

${ }^{16}$ A. Schmalz, et al., Phys. Rev. Lett. 67, 2854 (1991).

17 J. Neugebauer and M. Scheffler, Phys. Rev. B 46, 16067 (1992); Phys. Rev. Lett. 71, 577 (1993).

18 J. Burchhard, et al., Phys. Rev. B 50, 4718 (1994).

${ }^{19}$ C. Stampfl, et al., Phys. Rev. Lett. 69, 1532 (1992); Phys. Rev. B 49, 4959 (1994).

20 J. N. Andersen, E. Lundgren, R. Nyholm, and M. Qvarford, Phys. Rev. B 46, 12784 (1992).

${ }^{21}$ M. M. Nielsen, et al., Phys. Rev. Lett. 72, 3370 (1994).

${ }^{22}$ V. Gorodetskii, J. Lauterbach, H.-H. Rotermund, J. H. Block, and G. Ertl, Nature 370, 276 (1994).

${ }^{23}$ Z. C. Ying, J. Wang, G. Andronica, J.-Q. Yao, and E. W. Plummer, J. Vac. Sci. Technol. A 11, 2255 (1993). 
